\title{
SWFAD-IOT: Sistema de Apoyo a la Docencia en un Entorno IoT
}

\section{SWFAD-IOT: System Support for Teaching in an IoT Environment}

\author{
Gonzalo Cerruela García ${ }^{1}$, Nicolás García-Pedrajas², Irene Luque Ruiz³ ${ }^{3}$ Miguel \\ A. Gómez-Nieto ${ }^{4}$,
}

Fecha de recepción: 30/09/2018; Fecha de revisión: 05/04/2019; Fecha de aceptación: 23/05/2019

Cómo citar este artículo:

Cerruela García, G., García-Pedrajas, N., Luque Ruiz, I. \& Gómez-Nieto, M.A. (2019). SWFAD-IOT: Sistema de Apoyo a la Docencia en un Entorno IoT. Revista de Innovación y Buenas Prácticas Docentes, 8(2), 3444.

Autor de Correspondencia: gcerruela@uco.es

\section{Resumen:}

El presente trabajo muestra el desarrollo e implementación de un sistema de apoyo a la docencia mediante la web física en un entorno del Internet de las Cosas (IOT). El sistema está orientado al desarrollo de actividades académicamente dirigidas que potencien el trabajo por competencias y al diseño de acciones destinadas a mejorar los procesos de evaluación, potenciando la evaluación continua y la autoevaluación de los alumnos. Con buenos resultados se implementaron varios casos de uso para evaluar el sistema, construyendo una aplicación software que permite una interacción natural del alumno con el entorno docente que le rodea.

Palabras clave: Beacons, loT, Innovación docente, Web Física.

\section{Abstract:}

The present work shows the design and implementation of a support system for teaching through the physical web in an environment of the Internet of Things (IOT). The system is oriented to the development of academically directed activities that promote the work by competences and the design of actions destined to improve the evaluation processes, promoting the continuous evaluation and the self-evaluation of the students. With good results, several use cases were implemented to evaluate the system, building a software application that allows a natural interaction of the student with the surrounding teaching environment. Key Words: Beacons, Educational Innovation, IoT, Physical Web.

\footnotetext{
1 Universidad de Córdoba (España), gcerruela@uco.es; CÓDIGO ORCID: 0000-0001-9140-3347

2 Universidad de Córdoba (España), npedrajas@uco.es; CÓDIGO ORCID: 0000-0002-4488-6849

3 Universidad de Córdoba (España), ma1lurui@uco.es; CÓDIGO ORCID: 0000-0003-2996-7429

4 Universidad de Córdoba (España), mangel@uco.es; CÓDIGO ORCID: 0000-0002-1946-5495
} 


\section{INTRODUCCIÓN}

Actualmente la Web se compone de más de un billón de sitios con los que diariamente tenemos que interactuar en la búsqueda de información mediante navegadores específicos. Acciones tan comunes en el ámbito docente como buscar información sobre un determinado concepto implica tener que abrir un navegador, introducir una cadena de caracteres y filtrar todos los resultados que nos devuelve dicho navegador.

Recientemente ha surgido un nuevo concepto denominado Web física (Namiot \& Sneps-Sneppe, 2015; PhysicalWeb, 2016; Sneps-Sneppe \& Namiot, 2016), su objetivo es hacer que la información contextual esté disponible para cualquier persona, en cualquier momento. La idea principal es poder realizar todas las tareas cotidianas dependiendo de los objetos físicos circundantes (entorno del Internet de las cosas, IoT).

El uso de loT en el desarrollo de sistemas para el apoyo a la docencia ha proliferado en los últimos años, por ejemplo, se han desarrollado nuevas propuestas para el acceso de los alumnos a las fuentes bibliográficas recomendadas por los profesores (Borrego-Jaraba, Cerruela García, Luque Ruiz, \& Gómez-Nieto, 2013), surgen nuevos campus universitarios inteligentes en entornos loT (Majeed \& Ali, 2018) $y$, se proponen nuevos modelos educativos orientados a la mejora de los procesos enseñanza-aprendizaje (Nehru \& Chakraborty, 2020). Un resumen sobre la aplicación del loT en educación se puede entornar en (Ramlowat \& Pattanayak, 2019).

El concepto de Web física se ha podido convertir en una realidad gracias al desarrollo de balizas inalámbricas (beacons) que pueden admitir protocolos estándares como Bluetooth Low Energy (BLE) (Bluetooth, 2016). Estos dispositivos han permitido que los objetos del mundo real puedan mostrar información o servicios asociados mediante una URL.

A pesar del corto período de tiempo trascurrido desde la aparición de la Web física, son varias las propuestas prácticas de su aplicación en living labs y en desarrollos en explotación. Por ejemplo, en (Namiot \& Sneps-Sneppe, 2015) se realiza una propuesta que implementa la Web física dentro de la Smart City, este trabajo analiza dos casos de uso: el primero está destinado a la navegación en los interiores de aeropuertos, el segundo, está destinado a la subscripción de los usuarios a canales de información de la Smart City. En (Kibria \& Chong, 2015) se estudia la interacción entre los dispositivos físicos y los móviles dentro del estándar de la Web física, extendiendo los servicios del loT a una ontología semántica de lo que se conoce como "Web de los Objetos" (WoO).

La propuesta presentada en este trabajo tiene como finalidad crear un conjunto de servicios destinados a: i) el desarrollo de actividades académicamente dirigidas que potencien el trabajo por competencias y la transferencia del conocimiento teórico a la práctica, ii) garantizar una interacción natural del alumno con los procesos de evaluación, potenciando sistemas de evaluación continua que garanticen una mayor objetividad.

El trabajo se ha organizado de la siguiente forma: la sección 2 define los objetivos del proyecto, las principales características de los dispositivos BLE y la arquitectura software diseñada e implementada en este trabajo, la sección 3 describe los resultados experimentales obtenidos, finalmente, en la sección 4 se resumen las principales conclusiones del trabajo.

\section{DESARROLLO DE LA EXPERIENCIA DE INNOVACIÓN}

El objetivo principal del presente trabajo es el análisis, diseño e implementación de un sistema de apoyo a la docencia mediante la Web física en un entorno loT. El sistema está orientado al desarrollo de actividades académicamente dirigidas que potencien el trabajo por competencias y la transferencia del conocimiento teórico a la práctica. Además, se han diseñado acciones destinadas a mejorar los procesos de evaluación, potenciando la evaluación continua y la autoevaluación de los alumnos. 


\subsection{LOS DISPOSITIVOS BLE (BEACONS)}

La tecnología BLE surge con el anuncio por parte de "Bluetooth Special Interest Group (SIG)" de la versión Bluetooth Core Specification 4.0 (Bluetooth, 2016), la que entre otros aspectos permite una conexión inalámbrica continua (Bluetooth BR/EDR), lo que lo hace ideal para usos como el streaming de audio. Por otra parte, también es posible una conexión usando ráfagas cortas de largo alcance (Bluetooth LE), haciéndola ideal para el Internet de las Cosas. De esta forma las aplicaciones no requieren una conexión continua, aunque sigan dependiendo de la duración de la batería del dispositivo móvil.

Los dispositivos BLE, comúnmente también llamados beacons, utilizan la tecnología Bluetooth 4.0 para transmitir datos de forma periódica en paquetes de información con un formato preestablecido. Cada tipo de beacon usa una especificación propia para dar significado a los paquetes de datos que envía, actualmente son dos los protocolos más utilizados: iBeacon, URI Beacons (Eddystone open beacon).

iBeacon es el protocolo propuesto por Apple (Apple, 2016) y fue la primera tecnología BLE beacon en salir a la luz pública, sirviendo como referente para el resto de protocolos existentes. iBeacon es un estándar propietario que sostiene un amplio ecosistema de productos BLE y recursos software para desarrolladores, orientados fundamentalmente a la comunidad Apple.

El formato iBeacon se divide en cinco grupos para estructurar la siguiente información: i) datos específicos para la conexión (iBeacon Prefix), ii) el identificador único del beacon (UUID) para identificar el propietario de un grupo de beacons, iii) la identificación del subconjunto de beacons dentro de un grupo (major number), iv) el identificador individual de cada beacon (minor number), v) un valor que indica la intensidad de la señal a un metro del dispositivo (TX power).

El modo habitual de interacción mediante este protocolo deberá realizar un proceso de búsqueda del UUID, el "major number" y el "minor number", usándolos como referencia para buscar la información relacionada con el beacon en una base de datos. El campo TX power se utiliza para determinar a qué distancia se encuentra el dispositivo móvil del beacon.

En 2014 Google lanzó el proyecto UriBeacon (Uribeacon, 2014) para investigar cómo se podía utilizar la tecnología BLE para compartir URLs. Después de varios meses y muchas contribuciones en el desarrollo de utilidades y aplicaciones móviles basadas en UriBeacon, actualmente este formato se ha convertido en una parte de un proyecto más amplio, conocido como Eddystone (Eddystone, 2016), un protocolo que define un formato de mensajes BLE para beacons.

Este formato codifica en los cuatro primeros bits de mayor orden del UUID tres tipos de marcos o modos de funcionamiento: i) Eddystone-UID que envía un identificador único de 16 bits del beacon, ii) Eddystone-URL que envía una URL utilizando un formato comprimido de notificación, iii) Eddystone-TLM para enviar información de telemetría sobre el beacon, por ejemplo, el nivel de la batería, la temperatura del dispositivo, etc.

En abril de 2016 salió a la luz pública un cuarto modo de funcionamiento, el Eddystone-EID (Eddystone-EID, 2016), en este modo se envía un identificador efímero cifrado, cambiando periódicamente su valor dentro de un rango que se establece durante el registro inicial del dispositivo.

Este protocolo presenta una gran ventaja respecto al iBeacon dado que además de transmitir secuencias únicas (UUID) que pueden ser interpretadas por algunas aplicaciones, emite información que puede ser interpretada de forma nativa por cualquier dispositivo móvil sin tener que tener instaladas aplicaciones específicas.

\subsection{ARQUITECTURA DEL SISTEMA}

La arquitectura del sistema (Figura 1) está fundamentada en el uso de dispositivos BLE (beacons) que transmiten la URL de un determinado servicio o Web de interés en una 
zona circundante determinada por el radio de alcance del dispositivo. Este enfoque de solo emisión evita un problema de complejidad $O(\mathrm{~nm})$, si consideramos un entorno con $n$ usuarios donde cada uno de ellos se conecta a los $m$ dispositivos existentes. La idea es obtener un modelo que se adapte al peor caso de funcionamiento, garantizando que cada dispositivo BLE pueda estar constantemente difundiendo información y cualquier número de terminales pueda obtener dicha información con el menor número de conflictos. Por otra parte, este modelo garantiza la privacidad de los usuarios que pueden circular libremente sin dejar huella, gracias a que los dispositivos de radiodifusión no tienen información de quién está escuchando.

La URL se almacena en el beacon utilizando el protocolo Eddystone-URL con un tamaño máximo de 16 bits, el pequeño espacio de almacenamiento está orientado a evitar la congestión del sistema. Sin embargo, esto constituye un problema para almacenar cualquier tipo de URL, siendo necesario utilizar dominios cortos o un sistema para acortar el contenido de la URL. Para acortar las URLs se pueden utilizar servicios clásicos como Google URL Shortener (goo.gl, 2016) o bitly (bitly.com, 2016) donde la URL no se puede modificar. Otras soluciones proponen incluir en el servidor un sistema propio para acortar las direcciones, permitiendo modificar las direcciones y un control total de la configuración. Para ello existen soluciones de dominio público como Yourls o Polr (Polr, 2016; Yourl, 2016).

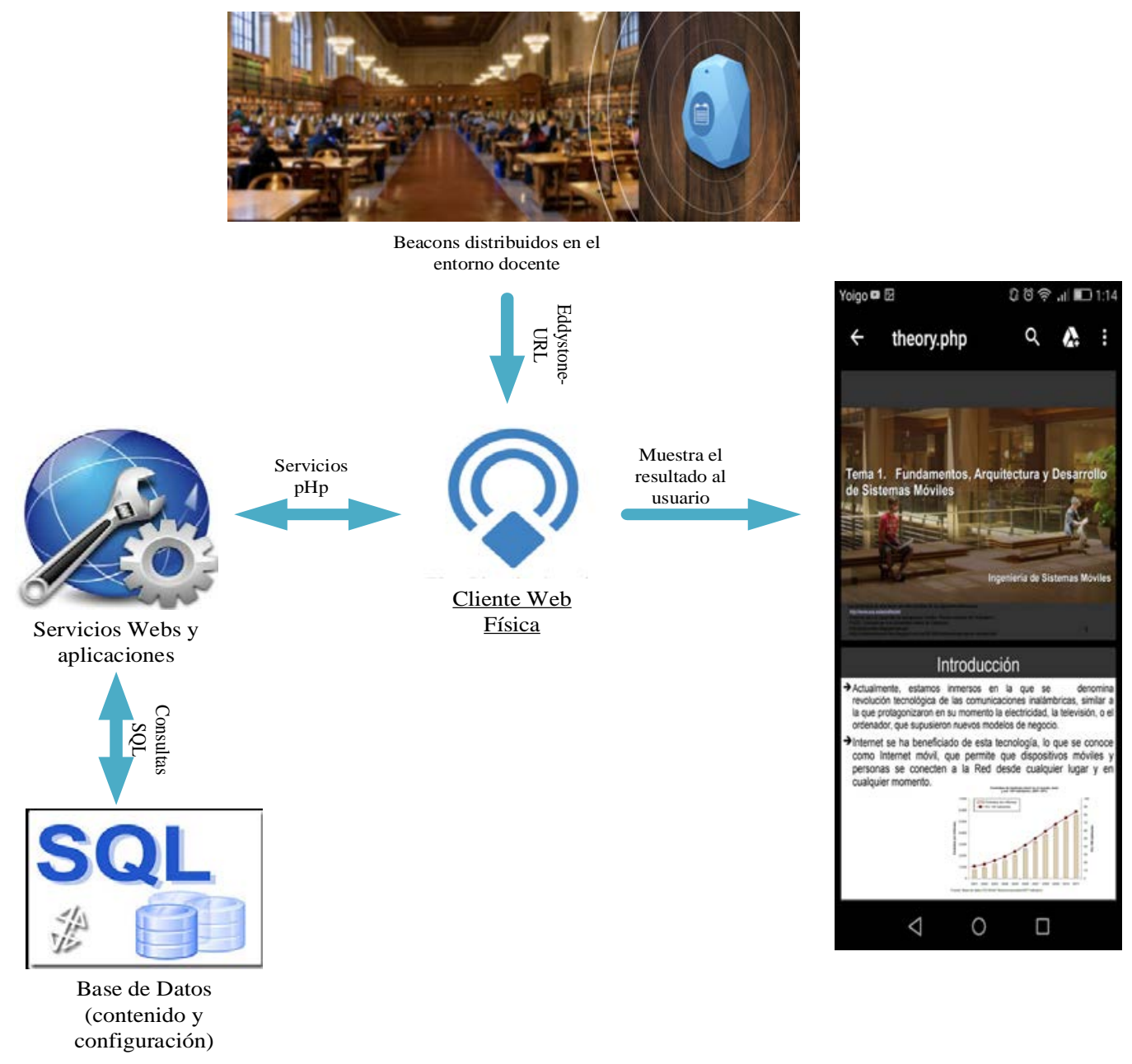

Figura 1. Arquitectura del sistema.

Como se muestra en la Figura 1 los beacons son distribuidos en un área de interés (facultad, aula, departamentos, despacho del profesor, etc.). Cada uno de estos dispositivos transmite la URL de un determinado servicio o aplicación. La arquitectura 
propuesta incluye un servidor web con el objetivo de lograr una completa personalización de estos servicios y aplicaciones.

Cada servicio puede estar asociado a uno o un conjunto de contenidos. Estos contenidos pueden ser de cualquier tipo: contenidos multimedia (texto, imágenes, videos, audios), enlaces Web, ficheros, etc., que a su vez pueden estar asociados a otros contenidos e incluso a otros servicios. Este modelo de servicios y contenidos y encapsulación de las aplicaciones en base a los mismos permite poder ofrecer cualquier tipo de solución a los usuarios finales que acceden a la URL almacenada en los beacons.

En los casos de uso implementados, dependiendo de su ubicación, los beacons tienen diferentes funciones tales como: i) notificación y acceso a información de interés relacionada con las asignaturas, por ejemplo, horario de clases y exámenes, resultado de las evaluaciones, noticias del entorno académico, etc. ii) notificaciones con información complementaria y/o actividades académicamente dirigidas sobre la clase de teoría o práctica a la que asiste el alumno. iii) Actividades evaluativas complementarias. No obstante, la solución creada permite implementar nuevos servicios asociados a los beacons de manera sencilla y fiable.

El servidor de aplicaciones/servicios web además de atender todas las solicitudes y personalizar los servicios, gestiona de forma flexible las direcciones URL almacenadas en los beacons. Cada beacon tiene una dirección fija, predefinida y acortada según las especificaciones Eddystone mediante el servicio URL Shortener de Google (goo.gl, 2016). Todas estas direcciones son interpretadas e identificadas por el servidor, garantizando la posibilidad de modificación y configuración, incluyendo la redirección a servicios externos. Los servicios externos serán todas las URL soportados por otros servidores de aplicaciones.

Como cliente para el acceso a los servicios de la Web física se aconseja la aplicación Chrome de Google por varias razones: i) está disponible para la mayoría de los dispositivos móviles, ii) de forma gratuita incluye el acceso a un servidor propietario de Google para verificar si las URLs cumplen las especificaciones de la Web física.

Para gestionar toda la información que requiere la plataforma de servicios de la Web física se diseñó e implementó un modelo de datos usando el sistema gestor de bases de datos PostgreSQL (PostgreSQL, 2019) que almacena información sobre:

- Los proyectos en los que se utilizarán los beacons. De esta forma se puede controlar y tener una realimentación del uso de la plataforma en diferentes experiencias docentes. Los proyectos tienen fecha de inicio y fin. Una vez finalizado el proyecto todos los servicios asignados al mismo no podrán ejecutarse.

- Todos los usuarios del sistema: administradores/responsables de los proyectos y usuarios terminales.

- Los beacons que se dan de alta en el sistema. Estos se identifican por un código interno y se indica la geolocalización en la cual se situará el beacon. Un beacon podrá tener asignado varios servicios, definiendo un atributo para determinar el orden de selección de esos servicios acorde a los siguientes criterios: a) Secuencial: se elegirán en orden de no usado o usado hace más tiempo. Si todos tienen la misma antigüedad se elige al primero según su código. b) Aleatorio: se eligen aleatoriamente. c) Todos: se ejecutará un servicio tras de otro.

- Los servicios que pueden ser ejecutados cuando se interacciona con un beacon. Cada servicio tiene asociada una plantilla (que puede variar) y un contenido (que también puede variar), su función es generar una página o interfaz con ese contenido, formateado con esa plantilla en el navegador del usuario.

- Los servicios son de tres tipos: a) Interno: se ejecuta una funcionalidad por el servidor. b) Externo: el servidor sólo es una pasarela a una web externa. c) Combinado: Si no se puede ejecutar el procedimiento interno se ejecuta el externo. Mediante atributos específicos se define si es obligado o no el inicio de 
sesión del usuario para que se ejecute el procedimiento interno, si es obligatorio conocer la geolocalización del usuario para que se ejecute el procedimiento interno, o para indicar la fecha en que cada servicio ha sido ejecutado por última vez.

- Los servicios asociados a cada beacon. De esta forma un beacon puede dar diferentes servicios de uno o diferentes proyectos.

- Los contenidos y las plantillas que definen cómo serán construidos los servicios. Las plantillas mantienen información de los diferentes formatos con los que se puede crear la interfaz para ser mostrada al usuario. Los contenidos será la información utilizada para generar la interfaz en base a una determinada plantilla.

- Por último, la base de datos almacena información histórica de los servicios ejecutados, sus contenidos y formato, de los usuarios que interaccionan con los beacons, etc. Esta información permitirá evaluar y realizar un estudio de usabilidad cuando la plataforma esté en fase de explotación.

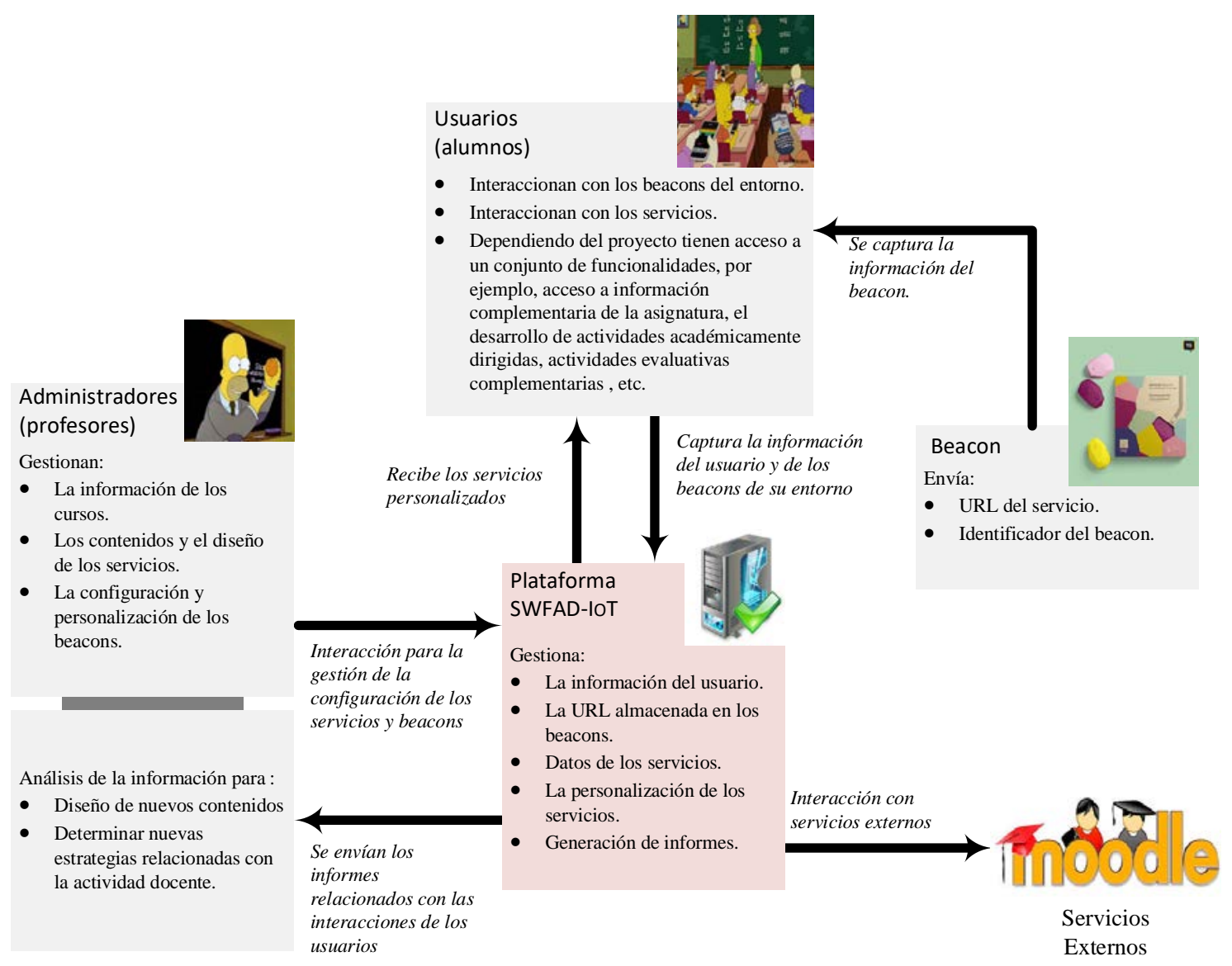

Figura 2. Modelo de interacción del Sistema.

La Figura 2 muestra el diagrama de interacción del sistema desarrollado. Para una determinada experiencia docente (proyecto) el perfil de administrador comienza el flujo de interacción insertando toda la información del proyecto, diseñando los contenidos y configurando todos los servicios.

La configuración de los servicios incluye la distribución georreferenciada de los beacons en los lugares de interés relacionados con el proyecto, su vinculación con los servicios y los períodos de su activación y desactivación. Esta información puede ser modificada en cualquier momento desde las opciones de administración que se habilitan para el tipo de usuario administrador. 
Los usuarios (alumnos) interaccionan con el sistema de forma natural según se van acercando a los objetos o lugares en los que se ha decidido colocar los beacons, recibiendo en su dispositivo móvil las notificaciones de todos los servicios disponibles. Estas notificaciones estarán ordenadas en función de la cercanía con el emisor.

Dependiendo de las características del proyecto, los usuarios pueden recibir servicios personalizados generados por la plataforma o la redirección a servicios soportados por agentes externos, por ejemplo, los contenidos y actividades docentes de la plataforma Moodle, la planificación docente, el horario de autobuses, etc.

En la interacción con los servicios los usuarios tendrán acceso a:

- Obtener información general y particular de la actividad docente.

- Recibir noticias en tiempo real de carácter general o relacionado con la actividad docente.

- Descarga de documentos relacionados con la actividad docente.

- Desarrollo de actividades académicamente dirigidas.

- Desarrollo de actividades evaluativas complementarias

- Obtener información complementaria dependiendo del lugar donde se realiza la actividad.

Todas las interacciones del usuario se almacenan en la base de datos, esta información es utilizada por los profesores responsables para determinar nuevas estrategias relacionadas con la asignatura, medir el aprendizaje de los alumnos, y poder evaluar el impacto del uso de la herramienta en el desarrollo de la actividad docente.

\section{RESULTADOS}

Para probar y evaluar el funcionamiento del sistema se eligió el campus de Rabanales de la Universidad de Córdoba, en este entorno docente se distribuyeron convenientemente un grupo de beacons que implementan los siguientes casos de uso: i) desarrollo de actividades académicamente dirigidas, ii) envío de noticias relacionadas con el entorno docente, iii) acceso a información complementaria relacionada con la actividad docente, iv) desarrollo de actividades evaluativas complementarias, v) acceso a servicios externos cercanos al lugar donde se desarrolla la docencia. Las Figuras 3-7 muestran algunas capturas de pantalla de la aplicación.

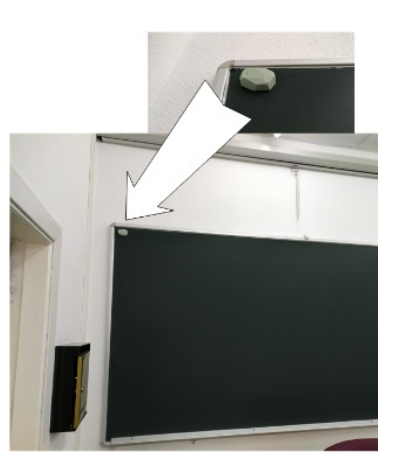

a)

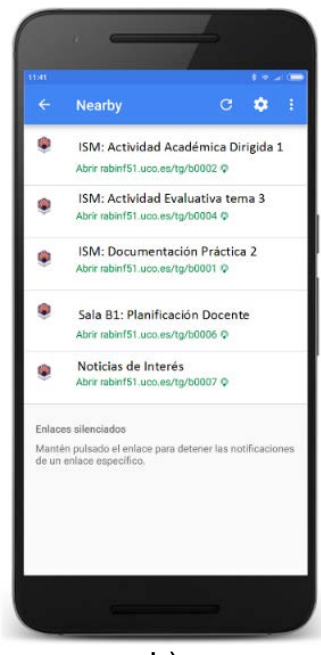

b)

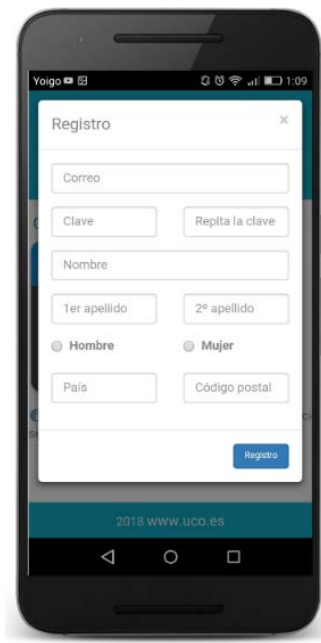

c)

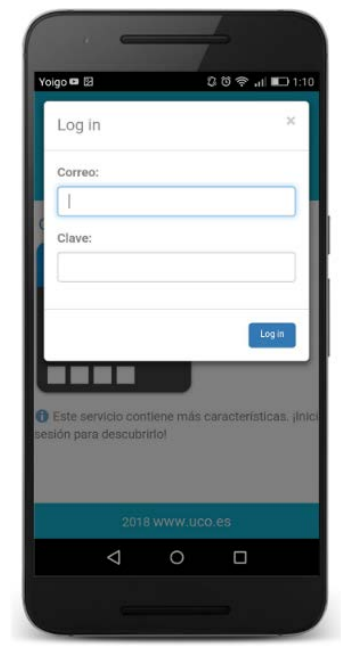

d)

Figura 3. Entorno loT, acceso al sistema. 
La interacción con los objetos o lugares de interés se realiza a medida que el usuario se aproxima a los beacons distribuidos estratégicamente para este fin, la Figura 3 a muestra un beacon ubicado en un aula del campus de Rabanales. La notificación (Figura $3 \mathrm{~b}$ ) incluye un mensaje con el formato establecido por la Web física y que incluye el icono de la web, una descripción y un enlace cuya función es la de disparar la aplicación y/o visualizar los contenidos generados por el propietario del servicio asociado. Como se muestra la Figura $3 \mathrm{~b}$, cuando hay varios dispositivos BLE emitiendo las notificaciones se ordenan según la proximidad del beacon al dispositivo móvil.

Para acceder al contenido de la notificación el usuario sólo tiene que tocar sobre la correspondiente notificación. Existen servicios que por seguridad requieren la autentificación y registro previo (Figura $3 \mathrm{c}$, d), en otros casos este paso no es necesario accediendo directamente al servicio al tocar sobre la notificación.

La Figura 4 (a-d) muestra el desarrollo de una actividad académicamente dirigida, en este caso se utilizan tres beacons ubicados en tres posiciones del aula distantes entre sí, los dispositivos permiten el acceso a diferentes contenidos sobre un mismo tema de interés. Los asistentes a la actividad son divididos en tres grupos ubicados convenientemente a un metro de distancia de cada beacon. De esta forma se da inicio al siguiente protocolo para la actividad:

- Los miembros del grupo, haciendo uso de sus dispositivos móviles, deberán realizar una lectura comprensiva de la información que pueden acceder desde la notificación más cercana.

- Pasado un período de tiempo, cada grupo realiza un debate interno sobre los contenidos estudiados.

- Finalmente, se elige un representante por grupo que realiza una exposición del tema para todos los alumnos. En este paso, se establece un debate en el cual participa el profesor corrigiendo las exposiciones y aportando información adicional de vital importancia.

Este servicio requiere el inicio de sesión de los alumnos, de esta forma se aprovecha la interacción para el control de la asistencia a la actividad. Para evitar un uso fraudulento, el sistema comprueba que la localización del alumno coincida con la ubicación del beacon.

La Figura $5(\mathrm{a}, \mathrm{b})$ muestra el caso de uso sobre el acceso a información complementaria relacionada con la actividad docente. En cada clase de teoría o práctica este servicio permite proporcionar a los alumnos información adicional sobre el tema impartido, normalmente esta información no suele estar publicada en Moodle, potenciando de esta forma la asistencia a clase. Para controlar la asistencia este servicio requiere el inicio de sesión de los alumnos.

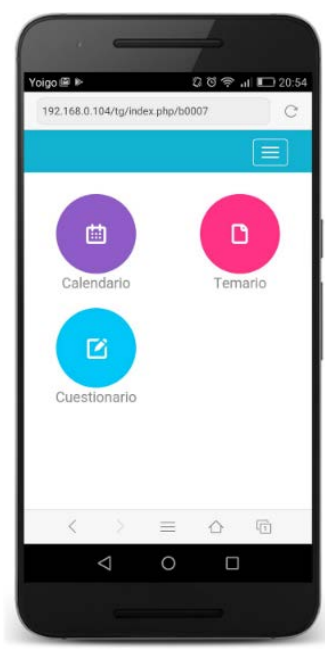

a)

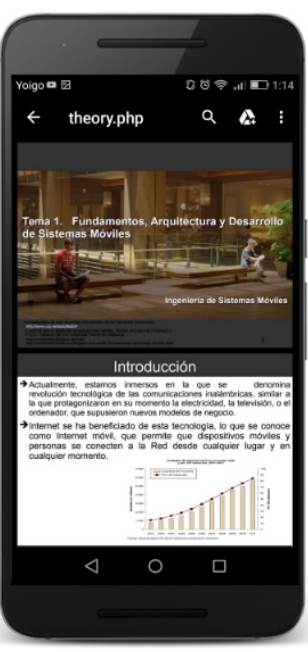

b)

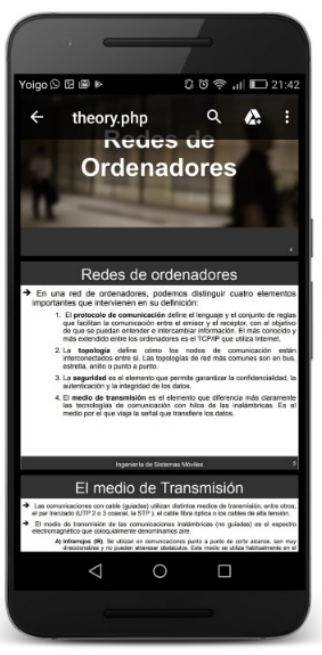

c)

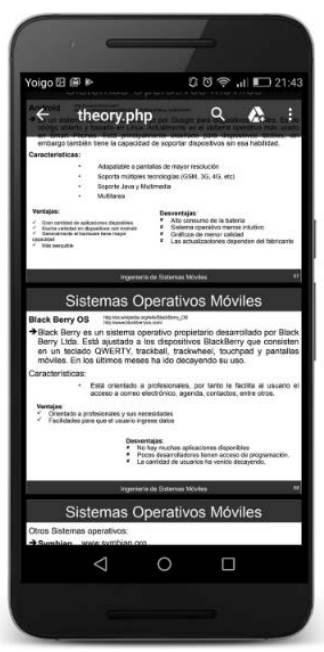

d)

Figura 4. Actividades académicamente dirigidas. 
La Figura 5 (c, d) muestra el resultado del caso de uso correspondiente a la distribución de noticias relacionadas con el entorno docente. Este servicio emite notificaciones de noticias de interés, es posible configurar la frecuencia de actualización, el número de noticias emitidas, las categorías, etc. Este servicio no requiere el inicio de sesión de los alumnos.

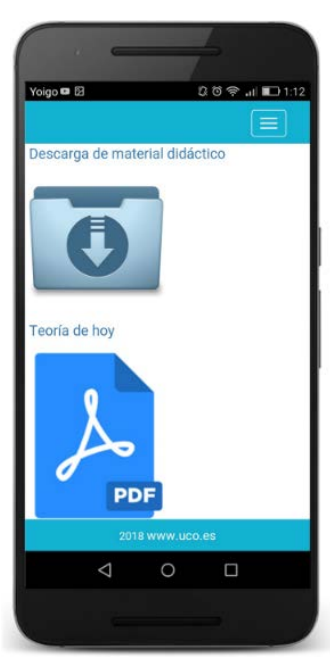

a)

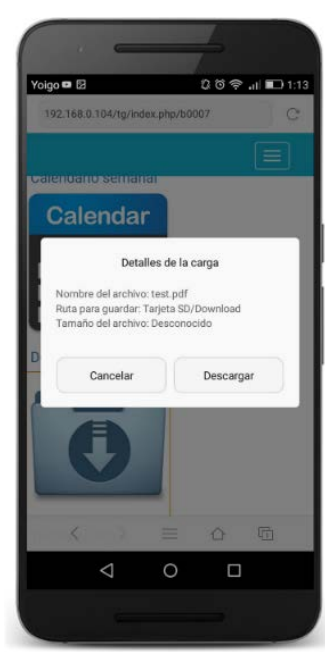

b)

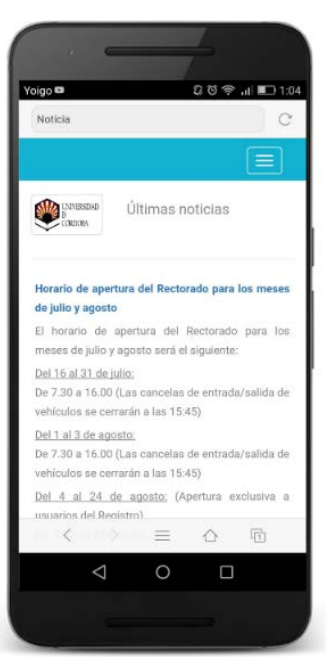

c)

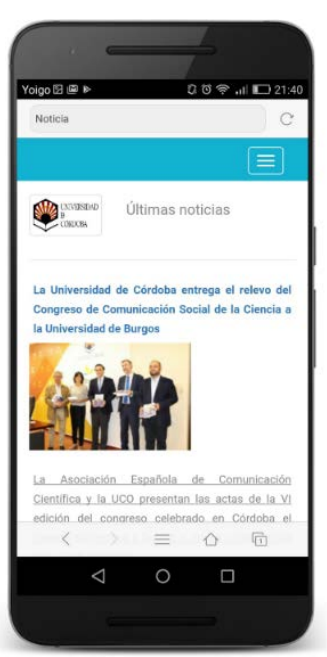

d)

Figura 5. Acceso a información complementaria (a, b), noticias del entorno docente (c, d).

La Figura 6 muestra los resultados para el caso de uso correspondiente al desarrollo de actividades evaluativas complementarias. Durante el desarrollo de una actividad docente, este servicio permite que los alumnos puedan realizar actividades orientadas a la autoevaluación o para complementar las evaluaciones de las convocatorias oficiales.

Como muestra la Figura 6 (a-d) el modelo de evaluación implementado corresponde a un cuestionario donde el alumno recibe la calificación automáticamente, la evaluación se puede repetir indefinidamente para motivar el autoaprendizaje, aunque a los efectos de la nota sólo se almacena la calificación inicial. Este servicio requiere el inicio de sesión de los alumnos, aprovechando la interacción para controlar la asistencia.

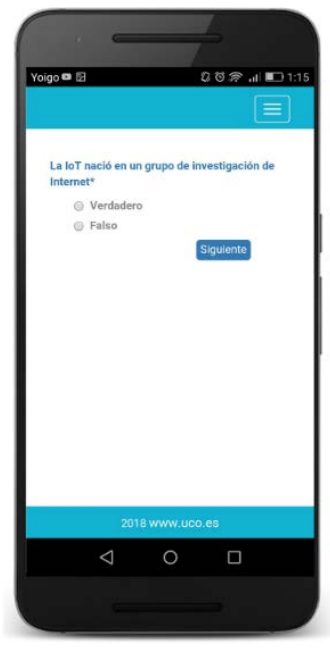

a)

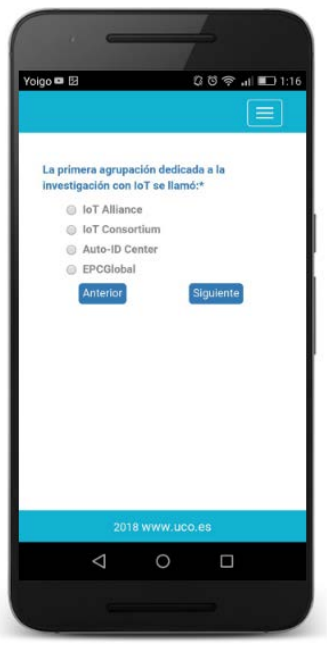

b)

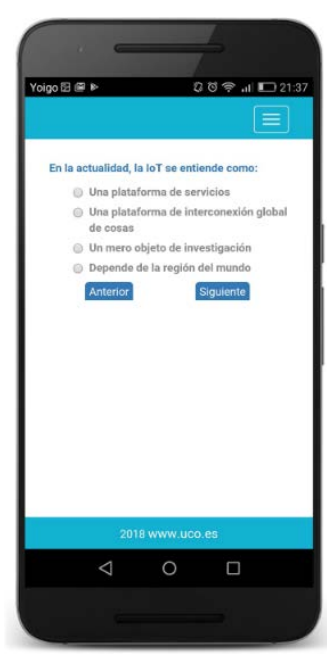

c)

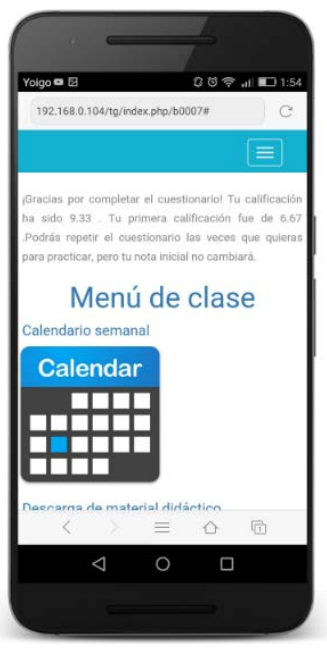

d)

Figura 6. Actividades evaluativas complementarias. 


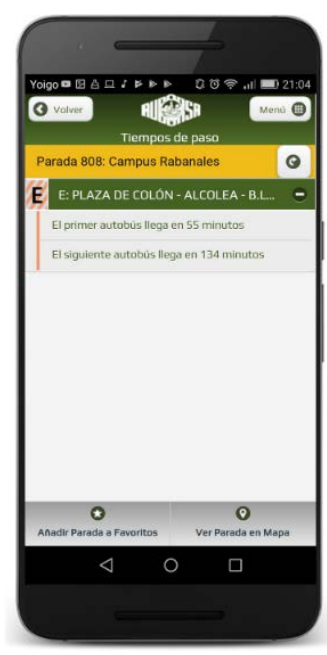

a)

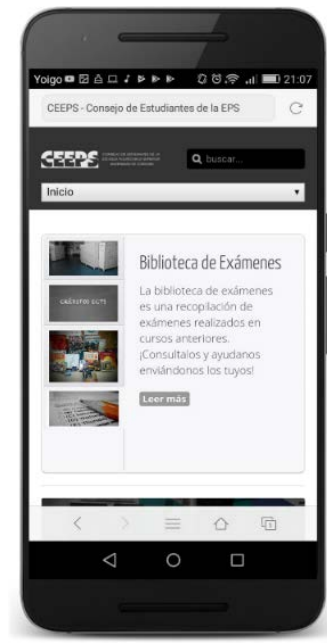

b)

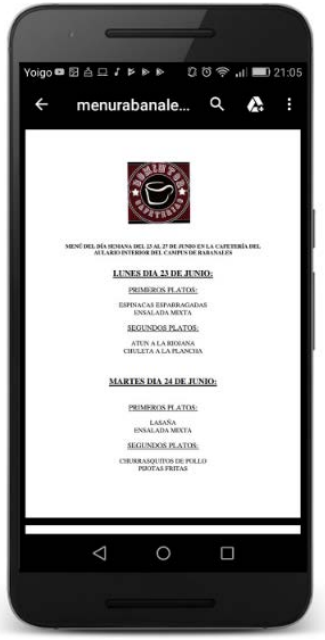

c)

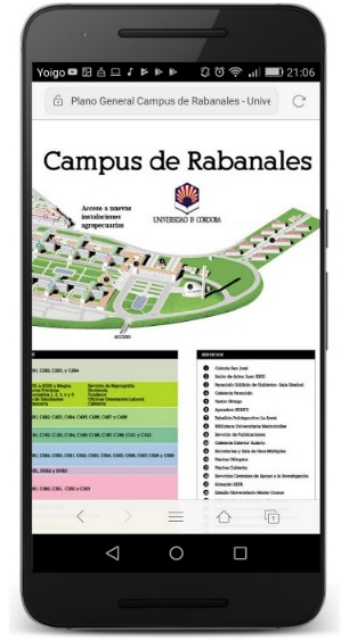

d)

Figura 7. Acceso a servicios externos.

La figura 7 muestra los resultados para el caso de uso correspondiente al acceso de servicios externos cercanos al lugar donde se desarrolla la docencia. Este tipo de interacción tiene como finalidad notificar sobre servicios externos que complementan la actividad docente. Por ejemplo, el acceso al horario de autobuses (Figura 7 a), información sobre fuentes externas que recopilan material docente (Figura 7 b), información gastronómica cercana al lugar donde se imparte la docencia (Figura 7 c) y, servicios que facilitan el desplazamiento en el entorno universitario (Figura $7 \mathrm{~d}$ ). Estos servicios no requieren el inicio de sesión de los alumnos.

\section{CONCLUSIONES}

El sistema propuesto en este trabajo cambia el modelo de interacción de los alumnos con los contenidos docentes, a diferencia de los actuales sistemas de virtualización en los que el alumno tiene que ir a la búsqueda de apuntes o actividades accediendo a una dirección web, nuestra iniciativa intenta traer los contenidos al contexto docente del alumno (aula, laboratorio de prácticas, objeto físico en estudio, etc.). Este nuevo sistema permite que el alumno interaccione de forma natural con los objetos que le rodean en lo que se conoce como el Internet de las Cosas (IoT).

La arquitectura software desarrollada permite personalizar los contenidos y los servicios para cualquier asignatura y/o entorno docente. Es posible configurarlo adaptándolo al comportamiento del alumno, para ello se hace uso de su interacción y del análisis del historial de su comportamiento.

Para la asignatura de Ingeniería de Sistemas Móviles del cuarto curso del grado en Ingeniería Informática el presente proyecto tiene un valor añadido, convertirse en un nuevo recurso docente y material de estudio de los últimos avances del loT aplicados a la innovación docente universitaria.

Actualmente el sistema es un prototipo en fase de pruebas, como línea de investigación futura se plantea solicitar un nuevo proyecto destinado a su implantación, evaluación/mejora, realizando además un estudio de usabilidad del sistema.

\section{AGRADECIMIENTOS}

A la Universidad de Córdoba por la financiación del Proyecto 2017-1-5005 en la convocatoria del Plan de Innovación y Buenas Prácticas Docentes 2017/2018. 


\section{REFERENCIAS}

Apple. (2016). ibeacon specifications. Recuperado de: https://developer.apple.com/ibeacon/.

bitly.com. (2016). Recuperado de: https://bitly.com/

Bluetooth. (2016). Bluetooth core specification. Recuperado de: https://www.bluetooth.com/specifications/bluetooth-core-specification.

Borrego-Jaraba, F., Cerruela García, G., Luque Ruiz, I., \& Gómez-Nieto, M. Á. (2013). An NFC based context-aware solution for access to bibliographic sources in university environments. Journal of Ambient Intelligence and Smart Environments, 5(1), 105-118.

Eddystone-EID. (2016). Eddystone Ephemeral Identifier. Recuperado de: https://developers.google.com/beacons/eddystone-eid (Accessed on jan 2016).

Eddystone. (2016). Eddystone specifications. Recuperado de: https://github.com/google/eddystone.

goo.gl. (2016). Recuperado de: https://goo.gl/

Kibria, M. G., \& Chong, I. (2015). Knowledge based open loT service provisioning through cooperation between physical web and WoO 2015 Seventh International Conference on Ubiquitous and Future Networks (pp. 395-400): IEEE.

Majeed, A., \& Ali, M. (2018, 8-10 Jan. 2018). How Internet-of-Things (IoT) making the university campuses smart? QA higher education (QAHE) perspective. Paper presented at the 2018 IEEE 8th Annual Computing and Communication Workshop and Conference (CCWC).

Namiot, D., \& Sneps-Sneppe, M. (2015). The Physical Web in Smart Cities. Paper presented at the Advances in Wireless and Optical Communications (RTUWO), 2015.

Nehru, R. S. S., \& Chakraborty, S. (2020, 2020//). The Education of Things (EoT) for Smart Learning Through IoT Intervention: A Case Study Based Analysis. Paper presented at the ICICCT 2019 - System Reliability, Quality Control, Safety, Maintenance and Management, Singapore.

PhysicalWeb. (2016). Recuperado de: https://google.github.io/physical-web/

Polr. (2016). Recuperado de: https://project.polr.me/

PostgreSQL. (2019). PostgreSQL Data Base. Recuperado de: https://www.postgresql.org/

Ramlowat, D. D., \& Pattanayak, B. K. (2019, 2019//). Exploring the Internet of Things (IoT) in Education: A Review. Paper presented at the Information Systems Design and Intelligent Applications, Singapore.

Sneps-Sneppe, M., \& Namiot, D. (2016). On physical web models. Paper presented at the Control and Communications (SIBCON), 2016 International Siberian Conference on.

Uribeacon. (2014). URI Beacon specifications. Recuperado de: https://github.com/google/uribeacon.

Yourl. (2016). Recuperado de: https://yourls.org/ 